\title{
Biomechanical implications of skeletal muscle hypertrophy and atrophy: A musculoskeletal model
}

Andrew D Vigotsky, Bret Contreras, Chris Beardsley

Muscle hypertrophy and atrophy occur frequently as a result of mechanical loading or unloading, with implications for clinical, general, and athletic populations. The effects of muscle hypertrophy and atrophy on force production and joint moments have been previously described. However, there is a paucity of research showing how hypertrophy and atrophy may affect moment arm (MA) lengths. The purpose of this model was to describe the mathematical relationship between the anatomical cross-sectional area (ACSA) of a muscle and its MA length. In the model, the ACSAs of the biceps brachii and brachialis were altered to hypertrophy up to twice their original size and to atrophy to onehalf of their original size. The change in MA length was found to be proportional to the arcsine of the square root of the change in ACSA. This change in MA length may be a small but important contributor to strength, especially in sports that require large joint moments at slow joint angular velocities, such as powerlifting. The paradoxical implications of the increase in MA are discussed, as physiological factors influencing muscle contraction velocity appear to favor a smaller MA length for high velocity movements but a larger muscle MA length for low velocity, high force movements. 
1 Biomechanical implications of skeletal muscle hypertrophy and atrophy:

a musculoskeletal model

5 Andrew D. Vigotsky ${ }^{1,2}$, Bret Contreras ${ }^{3}$, Chris Beardsley 4

6

$7 \quad{ }^{1}$ Kinesiology Program, Arizona State University, Phoenix, AZ

$8 \quad{ }^{2}$ Leon Root, M.D. Motion Analysis Laboratory, Department of Rehabilitation, Hospital for

9 Special Surgery, New York, NY

$10{ }^{3}$ School of Sport and Recreation, Auckland University of Technology, Auckland, New Zealand

$11{ }^{4}$ Strength and Conditioning Research Limited, London, UK

12

$13 *$ Corresponding author

14 E-mail: avigotsky@gmail.com 


\section{Introduction}

16 Muscle hypertrophy is a common adaptation to mechanical loading, typically delivered in the

17 form of long-term programs of resistance training. Muscle atrophy is a response to disuse that

18 occurs quickly following even short periods of mechanical unloading, which can be as little as

19 one week of strict bed rest (Dirks et al. 2015). Both muscle hypertrophy and atrophy have

20 important implications for clinical, general, elderly and athletic populations, because of the

21 relationship between measures of muscle mass or size and a range of performance and health

22 outcomes. For example, among strength athletes, measures of muscle mass or size have been

23 found to be very good predictors of Olympic weightlifting, powerlifting, and strongman

24 performance (Brechue \& Abe 2002; Siahkouhian \& Hedayatneja 2010; Winwood et al. 2012). In

25 the elderly, Janssen et al. (2004) found that low levels of muscle mass were strongly correlated

26 with an increased risk of disability, Malkov et al. (2015) reported that reducing thigh muscle size

27 was associated with an increased risk of hip fracture, and Srikanthan \& Karlamangla (2014)

28 found that low levels of muscle mass were associated with increased all-cause mortality. In

29 various clinical populations, reports have been made of increasing mortality or re-hospitalization

30 rates in individuals with lower levels of muscle mass (Greening et al. 2014; Streja et al. 2011;

31 Weijs et al. 2014).

32

33 The relationships between muscle size, or more accurately muscle cross-sectional area (CSA),

34 and measures of performance or disability are underpinned by the unique ability of muscle to

35 produce force, with greater muscle CSA corresponding to greater force production. At the

36 individual muscle level, force is produced by the contractions of single muscle fibers, which are 
37 collected together in groups, known as fascicles. Muscle CSA is measured in two ways:

38 anatomical CSA (ACSA) and physiological CSA (PCSA). ACSA is the muscle CSA measured

39 in the plane perpendicular to its tendons (the longitudinal axis), commonly recorded at the widest

40 point along the muscle. PCSA is the muscle CSA measured in parallel with the muscle fascicles,

41 which can vary for different parts of a pennate muscle. The angle between the longitudinal axis

42 and the direction of the muscle fascicles is the fascicle pennation angle $\left(\theta_{\mathrm{p}}\right)$. The component of

43 force exerted by the muscle fascicles is $\cos \theta_{\mathrm{p}}$ and therefore as $\theta_{\mathrm{p}}$ increases, this leads to a

44 progressively smaller component of muscle force being transmitted along the longitudinal axis to

45 the tendons. Consequently, where changes in muscle PCSA occur following a period of either

46 mechanical loading or unloading, the effect on muscle force transmitted to the tendons will also

47 be affected by any simultaneous changes in $\theta_{\mathrm{p}}$, with the increases that occur following

48 mechanical loading reducing the beneficial effect of hypertrophy, and the decreases that occur

49 following unloading mitigating some of the adverse effects of atrophy (Aagaard et al. 2001;

50 Erskine et al. 2014; Kawakami et al. 1995; Seynnes et al. 2006). In order to accurately predict

51 joint moments, it is important for musculoskeletal models to take into account all of the

52 aforementioned architectural variables.

54 Such changes in muscle PCSA, ACSA, $\theta_{\mathrm{p}}$, and fascicle length that follow from exposure to 55 mechanical loading have been well documented (Aagaard et al. 2001; Erskine et al. 2014;

56 Kawakami et al. 1995; Seynnes et al. 2006). The changes that simultaneously occur in various

57 measures of strength are often attributed to these architectural and morphological changes, in

58 addition to neural adaptations (Behm 1995). Yet, the change in muscle size appears to be one of

59 the most important, if not the most important factor: Erskine et al. (2014) reported that changes 
60 in both muscle volume and muscle ACSA were strongly associated with increases in maximum

61 voluntary isometric contraction (MVIC) force following a 12-week period of elbow flexor

62 resistance training. Although the MVIC force values reported by Erskine et al. (2014) were

63 recorded using a linear force transducer, they are actually indicative of a net joint moment.

64 Nevertheless, it is important to note that measures of MVIC force recorded externally using load

65 cells (as in Erskine et al. (2014)) are not identical to the isometric muscle forces produced

66 internally, as the external force reflects a net joint moment. Internal isometric muscle force is a

67 product of the single muscle fiber force (also called specific tension), PCSA and $\theta_{\mathrm{p}}$. The internal

68 isometric muscle force is transmitted along the longitudinal axis of the muscle through the

69 tendons to act on the bones either side of a joint, where it creates an isometric moment about the

70 joint. This isometric joint moment is the product of the internal isometric muscle force and the

71 moment arm (MA) length. The MA length is the perpendicular distance between the muscle line

72 of action to the joint center of rotation. Consequently, changes in both internal isometric muscle

73 force and MA length can affect the magnitude of the maximum isometric joint moment that can

74 be produced.

76 Although muscle ACSA appears to be a key determinant of MVIC moment production, the

77 changes in MA length that occur as a result of either hypertrophy or atrophy have been less well

78 described. Nevertheless, it appears that there is a relationship between agonist muscle size and

79 the MA associated with the joint action. Sugisaki et al. (2010) and Akagi et al. (2015) described

80 a positive correlation between muscle size and muscle MA length. These findings suggest that

81 larger muscles are likely to benefit from a longer MA and consequently that hypertrophy may

82 lead to increases in MA length and atrophy to decreases in MA length, which may have impacts 
83 on the joint moment that go above and beyond alterations in muscle force. Indeed, Sugisaki et al.

84 (2014) noted a small increase in triceps brachii MA length following hypertrophy of around

$855.5 \%$, albeit which they deemed to be small and possibly negligible.

86

87 Since the relationship between MA length and muscle size is not fully understood, the purpose of

88 this paper is to develop a two-dimensional mathematical model to describe how changes in

89 ACSA of the proximal elbow flexors change MA length, $\theta_{\mathrm{p}}$, and joint moment contributions.

90 Materials and Methods

91

92 A mathematical model that related the ACSA of both BIC and BRA to elbow flexion joint

93 moment contributions was created using WolframAlpha (Wolfram Research, Champaign,

94 Illinois) and Excel (Microsoft, Seattle, USA), and the regression was tested in Excel and Stata

95 (StataCorp, College Station, TX).

Development of the Musculotendinous Unit

98 A position-elbow flexor ACSA hyperbolic cosine regression equation was extrapolated from

99 West et al. (2010), wherein magnetic resonance images (MRIs) were taken of the elbows of 65

100 subjects, from $2.4-11.2 \mathrm{~cm}$ proximal to the elbow, in both elbow extension and in a neutral

101 radioulnar joint position.

102

103 Let $x$ be distance from the elbow, from distal to proximal, in centimeters $(\mathrm{cm})$. Let 104 


$$
\operatorname{ACSA}(x)=-\cosh (0.48(x-7.3))+23
$$

106

107 Therefore, the radius, assumed to be the centroid and average line of pull of the proximal elbow

108 flexors, assumed to be a cylinder (van der Linden et al. 1998), can be defined as a function of

$109 A C S A(x)$ :

110

$$
r(x)=\sqrt{\frac{A C S A(x)}{\pi}}
$$

113 Let $\alpha=$ coefficient of hypertrophy, or the degree to which the muscle is hypertrophying (or

114 atrophying) from baseline, which assumes uniform growth across the muscle belly.

115

116 Let $\beta=$ the $x$ position of the musculotendinous (MT) junction. The centroids of the distal BIC

117 and BRA tendons, tangent to the centroid of the distal-most section of each muscle belly $(t)$,

118 assumed to be the line of pull, can be described as:

119

$$
t(x, \alpha, \beta, \varphi)=\frac{d r}{d x}(\beta) x+\varphi \sqrt{\alpha} \mathrm{r}(\beta)-\beta \frac{d r}{d x}(\beta)
$$

122 where $\varphi$ is a constant to correct for muscle location. More specifically, it was assumed that the

123 BIC lay directly over (superficial and anterior to) the BRA. Because Equation 1 represents the

124 ACSA of both the BIC and BRA, the centroid of this (Eq. 2) represents the division between the

125 BIC and BRA, under the assumption that the muscles are equal in size. Therefore, when $\varphi=0.5$,

126 that represents half of the position of the centroid derived from Equation 1, and when $\varphi=1.5$, 
127 that represents one and a half times the position of the centroid derived from Equation 1. Under

128 the aforementioned assumptions, the former would represent the BRA, and the latter represents

129 the BIC. The Cartesian outcomes of $t$ and $r$ in context of the model are illustrated in Figure 1.

130 The mathematical definitions of $B I C$ and $B R A$ depicted in Figure 1 are described in the

131 proceeding paragraphs.

132

133 [ Insert Figure 1 about here. ]

135 Because $r(x)$ represents the centroid of both the BIC and BRA, it is necessary to divide this into 136 each individual muscle. Under the assumption that the muscle belly of the BIC was set to begin

$1371.1 \mathrm{~cm}$ proximal to the joint center in order to control for insertion point, which was fixed 4.51

$138 \mathrm{~cm}$ distal to the axis of rotation (capitulum). This was assumed to be approximately the center of

139 the insertion site, as the capitulum has a $1.06 \mathrm{~cm}$ radius (Shiba et al. 1987), the bicipital

140 tuberosity is $2.5 \mathrm{~cm}$ distal from the radial head, and the insertion site is $2.2 \mathrm{~cm}$ long (Mazzocca et

141 al. 2006). As per the MRIs, the muscle belly was set to end $11.2 \mathrm{~cm}$ proximal to the elbow joint.

142 Therefore, the BIC MT unit (MTU) can be described as:

143

144

$$
\operatorname{BIC}(x, \alpha)= \begin{cases}\mathrm{t}\left(x, \alpha, 1.1, \frac{3}{2}\right) & x<1.1 \\ \frac{3}{2} \sqrt{\alpha} \mathrm{r}(x) & 1.1 \leq x \leq 11.2\end{cases}
$$

146 The muscle belly of the BRA was set to begin $0.69 \mathrm{~cm}$ proximal to the joint center in order to

147 control for insertion point, which was fixed $3.17 \mathrm{~cm}$ distal to the axis of rotation (trochlea). Like

148 the BIC, it was assumed that this was the center of the insertion site, as the trochlea has a 0.75 
$149 \mathrm{~cm}$ radius (Murray et al. 2002), the coronoid process is about $1.10 \mathrm{~cm}$ from the trochlea, and the

150 insertion site is about $2.63 \mathrm{~cm}$ long (Cage et al. 1995). Therefore, the MTU of the BRA can be

151 described as

152

$$
\operatorname{BRA}(x, \alpha)= \begin{cases}\mathrm{t}\left(x, \alpha, 0.69, \frac{1}{2}\right) & x<0.69 \\ \frac{1}{2} \sqrt{\alpha} \mathrm{r}(x) & 0.69 \leq x \leq 11.2\end{cases}
$$

155 Both the BIC and BRA were modeled on the interval $0.5 \leq \alpha \leq 2.0$, with a step of 0.1 .

Moment Arm, Force, Pennation Angle, and Normalized Muscle Force Calculations

158 The joint center of the elbow is represented by the origin $(0,0)$, and the perpendicular distance

159 from the tendon to the joint center was then calculated as the MA. This was done so by finding

160 the angle of insertion via arctangent and using that angle to find the vertical, or perpendicular,

161 component by multiplying the lever arm by the sine of the insertion angle (Eq. 6).

162

163

$$
M A=\gamma \cdot \sin \left(\arctan \left(\frac{\partial(B I C \vee B R A)}{\partial x}(\beta, \alpha)\right)\right)
$$

164 where $\gamma$ is the muscle's insertion point.

165

Muscle and Tendon Forces

167 Increases in pennation angle were assumed to occur proportionally with increases in maximum

168 ACSA (Erskine et al. 2014). In order to calculate muscle force, normalized muscle force (NMF)

169 was assumed to be $30.75 \mathrm{~N} \cdot \mathrm{cm}^{-2}$, which is the average of previously reported values $(23,30,33$, 
170 and $\left.37 \mathrm{~N} \cdot \mathrm{cm}^{-2}\right)($ Edgerton et al. 1990; Ikai \& Fukunaga 1968; Nygaard et al. 1983; Ralston et al.

171 1949), and was assumed to remain constant with changes in ACSA. Muscle force $\left(F_{\text {muscle }}\right)$ was

172 derived from NMF by multiplying NMF by the ACSA (Eq. 6). The use of ACSA rather than

173 PCSA is appropriate in this context, as Kawakami et al. (1994) found no statistically significant

174 difference between ACSA and PCSA of the elbow flexors.

$$
F_{\text {muscle }}=A C S A \cdot N M F
$$

176 Tendon force $\left(F_{\text {tendon }}\right)$ was then calculated as the parallel component of the muscle's force (Eq. 177 8).

178

179

$$
F_{\text {tendon }}=F_{\text {muscle }} \cdot \cos \theta_{p}
$$

Moment Contributions

181 The elbow flexion moment contributions of the BIC and BRA $(M)$ were calculated by simply

182 multiplying each muscle's tendon force by its respective moment arm.

183

184

185

186

187 In order to validate the model, the primary outcomes - that is, MA length, pennation angle, and

188 joint moment trends - were compared with previous, relevant literature.

\section{Results}

190 Equation 1 showed a strong correlation with the length-ACSA relationship described by West et

191 al. (2009) $(\mathrm{p}<0.001 ; \mathrm{r}=0.911)$. The results of the model, including the effects of ACSA on

192 MA, elbow flexion moment contributions, pennation angle, muscle force, and tendon force, can 
193 be found in Figure 2. The key data for the atrophy, baseline and hypertrophy conditions are

194 shown in Table 1.

195

196 [ Insert Table 1 about here ]

197

198 [ Insert Figure 2 about here ]

199 BIC and BRA MA lengths increased approximately hyperbolically with increases in ACSA

200 (Figure 2A). Angle of pennation for both BIC and BRA increased linearly with increases in

201 ACSA (Figure 2B). Both the BIC and BRA were shown to have identical muscle forces (Figure

202 2C), but due to the differences in pennation angles (Figure 2B), the differences in tendon force

203 became more apparent with greater muscle ACSA (Figure 2D). Figure 2E depicts how changes

204 in MA length affect elbow flexion moment contributions, as the dashed lines illustrate the

205 moment contributions if the MA length did not change (remained identical to baseline (11.0

$\left.206 \mathrm{~cm}^{2}\right)$ ). At $22.0 \mathrm{~cm}^{2}$, accounting for changes in BIC and BRA MA length result in $27.2 \%$ and

$20737.3 \%$ greater joint moment contributions, respectively (Figure 2E).

\section{Discussion}

209 The MA lengths of the BIC and BRA at baseline in this model were within previously reported

210 ranges in some studies (An et al. 1981; Ramsay et al. 2009), and although they may appear

211 shorter than those reported by some other investigators (Amis et al. 1979; An et al. 1981;

212 Edgerton et al. 1990; Pauwels 1980), most studies did not report MA lengths in the same joint

213 positions utilized for this model. Furthermore, the MA lengths reported in this model are similar

214 to those previously modeled by Murray et al. (1995) (Figure 2A). The increases in pennation 
215 angles reflect values and trends previously reported, in that pennation angle increases linearly

216 with increases in ACSA (Erskine et al. 2014; Ikegawa et al. 2007; Kawakami et al. 1993;

217 Kawakami et al. 1995) (Figure 2B). Training studies corroborate the described increase in elbow

218 flexion moment contributions, as it has been shown that net elbow flexion moments increase

219 linearly with increases in ACSA(Erskine et al. 2010a; Erskine et al. 2010b).

220

221 To the authors' knowledge, this is the first model to describe the effects of muscle hypertrophy

222 and atrophy on MA length. It revealed important changes in MA lengths of the BIC and BRA

223 with increases in ACSA (Figure 2A, Figure 2E). Previous research has only attributed increases

224 in net joint moment production to the effects of hypertrophy on muscle force (Aagaard et al.

225 2001; Erskine et al. 2014), while ignoring potential changes in MA length, as described by our

226 model. Intuitively, this change in MA length must be a function of a change in insertion angle, as

227 the insertion point cannot shift. This increase in insertion angle occurs as the size of the muscle

228 belly increases, thereby shifting the resultant vector of the muscle further from the humerus and

229 consequently from the joint center. This is exemplified by the ACSA at baseline $\left(11.0 \mathrm{~cm}^{2}\right)$

230 corresponding to MA lengths of $0.75 \mathrm{~cm}$ and $2.17 \mathrm{~cm}$ for the BRA and BIC, respectively, while

231 the ACSA of a muscle double that size $\left(22.0 \mathrm{~cm}^{2}\right)$ corresponds to MA lengths of $1.03 \mathrm{~cm}$ and

$2322.76 \mathrm{~cm}$ for the BRA and BIC, respectively (Table 1, Figure 3). In other words, for a $100 \%$

233 increase in ACSA, the MA lengths of the BIC and BRA increase by $27.2 \%$ and $37.3 \%$,

234 respectively.

235

236 [ Insert Figure 3 about here ] 
238 The modeled change in MA length for the BIC and BRA in this particular joint position (i.e.,

239 neutral radiulnar joint in elbow extension) is proportional to the arcsine of the square root of the

240 change in ACSA $(\triangle M A \propto \arcsin (\sqrt{\triangle A C S A})$ ). This is due to lengths being proportional to the

241 square root of the area in which they are contained (as in Equation 2), while the arcsine controls

242 for the perpendicular component (as in Equation 6). The relationship observed by Sugisaki et al.

243 (2014) elucidates just how subtle changes in MA length can be, as a 33.6\% increase in triceps

244 brachii ACSA was accompanied by a 5.5\% increase in MA length. It is unclear if other muscles,

245 joints, or joint angles would behave similarly under hypertrophy, as these results cannot be

246 extrapolated, nor can it be said for certain whether this relationship would hold true for other

247 joint angles.

248

249 Although the increase in MA length appears to be beneficial for static or quasi-static strength, it 250 may be detrimental to high velocity, dynamic movements. This is paradoxical, because a larger

251 MA length should be helpful in producing a larger net joint moment, which might be expected to 252 increase angular acceleration, irrespective of joint angular velocity. However, owing to the 253 biomechanical properties of muscle, this is not the case. Specifically, in accordance with the

254 force-velocity hyperbolic relationship, less force can be developed during a high velocity, 255 dynamic muscle action than during a slow velocity or isometric muscle action (Hill 1938). It is 256 thought that the force-velocity relationship may arise as a result of a number of factors, including 257 the number of cross-bridges (Piazzesi et al. 2007), fluid friction or viscosity (Gasser \& Hill 1924; 258 Hill 1922), adenosine diphosphate (ADP) dissociation rates (Nyitrai et al. 2006), and passive 259 elastic tension at long lengths (Abbott \& Wilkie 1953). Irrespective of the underlying 260 mechanisms, however, Nagano \& Komura (2003) showed that a shorter MA length is beneficial 
261 for high velocity, dynamic muscle actions, since a given length change in the muscle will

262 necessarily cause greater joint excursion. Consequently, with a shorter MA length, more muscle

263 force can be produced, as a shorter change in length is required, which necessitates a smaller

264 contraction velocity. Mathematically, this can be described by the equation for MA length, where

$265 d l$ is the instantaneous change in MTU length and $d \theta$ is the instantaneous change in joint angular

266 displacement (in radians) (Eq. 8). With a larger MA length, a greater change in MTU length

267 must occur for the same change in joint angular displacement, which would require a greater

268 contraction velocity. The interactions of the ramifications of an increased MA on angular

269 velocity and angular acceleration require further investigation, and may have several, important

270 sport- or task-specific implications.

271

272

$$
M A=\frac{d l}{d \theta}
$$

\section{Limitations}

276 There were a number of limitations inherent in this study that arose from the assumptions that

277 were made. It was assumed that there was no change in either muscle fascicle or tendon length

278 during the production of the calculated contributory elbow flexion joint moments, but changes in

279 both muscle fascicle length and tendon elongation have been observed in vivo, in the tibialis

280 anterior (Ito et al. 1998). Similarly, it was assumed that no myofascial force transfer occurred

281 between the elbow flexors, although it has been observed that there are several ways in which

282 this could occur (Huijing \& Jaspers 2005). 
284 Our model assumed no changes in NMF, which directly affects muscle force calculations and

285 thus joint moment contributions. Although force production typically increases to a greater

286 extent than ACSA, some studies have reported reductions in normalized force following

287 resistance training, albeit not in the elbow flexors (Ikegawa et al. 2007; Kawakami et al. 1995;

288 Sale et al. 1992). When considering studies performed in the elbow flexors, it is, however,

289 apparent that normalized force typically either increases (Brandenburg \& Docherty 2002; Vikne

290 et al. 2006) or remains constant (Narici \& Kayser 1995; Takarada et al. 2000). These equivocal

291 findings indicate that our model may or may not reflect the typical changes in normalized force

292 expected during resistance training for the elbow flexors, which may therefore arise due to

293 central factors influencing strength gains or peripheral changes affecting single fiber specific

294 tension.

295

296 Indeed, although excluded in our assumptions, studies have shown increases in single fiber

297 specific tension using both in vivo (Erskine et al. 2011; Erskine et al. 2010a; Erskine et al.

298 2010b) and in vitro designs (Erskine et al. 2011; Pansarasa et al. 2009; Parente et al. 2008)

299 following resistance training protocols, which were not necessarily accompanied by muscle

300 hypertrophy. Changes in specific tension observed in vivo indicate that changes in either

301 extracellular lateral force transmission or myofibrillar packing density might be responsible for

302 alterations in normalized force, while changes observed in vitro can likely only be explained by

303 alterations in myofibrillar packing density. Additionally, it has been reported that changes in

304 specific tension occur in tandem with atrophy and that such changes appear to be associated with

305 reduced myofibrillar packing density (Riley et al. 1998). Whether the exact same mechanisms 
306 are involved in the alteration of specific tension with mechanical loading and unloading,

307 however, is unclear.

308

309 The use of a cylinder to model the BIC and BRA is not necessarily completely accurate, but

310 cylindrical shapes have been used in previous muscle models, such as the gastrocnemius (van der

311 Linden et al. 1998). However, notwithstanding this point, the same proposed mathematical

312 relationship would still stand with other, irregular shapes, as all linear distances within a cross-

313 section are related to the square root of the area of that cross-section.

314

315 Our model assumed that there were no changes in fascicle length, changes in fiber type area

316 proportion, or shifts in the fiber type of individual muscle fibers. However, previous research

317 indicates that changes do occur in fascicle length (Baroni et al. 2013; Blazevich et al. 2003;

318 Reeves et al. 2009) and in respect of proportional muscle fiber type areas (Campos et al. 2002;

319 Schuenke et al. 2012; Staron et al. 1991), although the literature is currently equivocal regarding

320 the exact nature of these responses to both resistance training and disuse atrophy (de Souza et al.

321 2014; Kawakami et al. 1995). In addition, the effect of muscle fiber type on maximal strength is

322 likely less important than its effect on dynamic strength, as the specific tension of type I and type

323 II muscle fibers is not normally found to be substantially different (e.g., Harber \& Trappe

324 (2008)) but there is often a marked difference reported in muscle contraction velocity (e.g.,

325 Harber \& Trappe (2008)). However, it can be argued that the model is more robust and free from

326 possible confounding factors because the aforementioned variables were not included, so they do

327 not have to be "teased out". 
329 Additionally, although excluded in our assumptions, changes in agonist voluntary activation or

330 co-contraction might be expected to occur following periods of mechanical loading or unloading.

331 Indeed, some studies have reported small increases in voluntary activation following resistance

332 training (Ekblom 2010; Erskine et al. 2010b), which may imply that mechanical loading involves

333 changes in neural drive. Whether such increases are likely to have a substantial impact on

334 strength gains given that voluntary activation levels of $>93 \%$ are frequently observed in young,

335 untrained subjects before commencing resistance training (Erskine et al. 2010b; Power et al.

336 2015; Venturelli et al. 2015) appears questionable. Nevertheless, there is some evidence that

337 voluntary activation is reduced in the elderly, possibly following sustained periods of disuse

338 leading to atrophy (Klass et al. 2007), and there is good evidence that such reductions in

339 voluntary activation can be reversed following sustained programs of resistance training (Arnold

$340 \&$ Bautmans 2014). The effect of changes in antagonist co-contraction following periods of

341 mechanical loading and unloading is much less clear. There does not appear to be any difference

342 between antagonist co-contraction activity between trained and untrained individuals (Maeo et

343 al. 2013), nor does antagonist co-contraction activity change following training in young (Maeo

344 et al. 2014) or old (Arnold \& Bautmans 2014) individuals. Like the changes in fascicle and fiber

345 characters, it can also be argued that by not including these neural adaptations, the model

346 includes less possible confounding variables. Therefore, the modeled changes in forces and

347 moments are strictly due to the architectural characteristics included in the model.

349 Lastly, and perhaps most importantly, this model only examined two muscles in one joint

350 position (i.e., neutral). It is likely that with changing joint positions, the relationship would shift

351 (Murray et al. 1995). For example, during elbow flexion, BIC and BRA MA lengths are greater 
352 because the insertion angle approaches $90^{\circ}$, so greater hypertrophy may be disadvantageous for

353 MA length in such positions, as it may shift the insert angle away from $90^{\circ}$. Such circumstances

354 have yet to be described and modeled, however.

\section{Conclusions}

356 The contribution of changes in ACSA to joint moment contributions following hypertrophy and

357 atrophy resulting from mechanical loading and unloading are not fully understood, nor are the

358 implications of concomitant changes in MA length. This model was the first to describe how

359 changes in ACSA following hypertrophy or atrophy of the BIC and BRA might alter MA length

360 and how both changes in ACSA and MA length might impact on relative elbow flexion moment

361 contributions in neutral radioulnar and elbow joint positions. The results of this model should be

362 interpreted with caution, as the predicted outcomes (namely, MA lengths) have not been

363 demonstrated in vivo. Additionally, only one joint position (neutral) was investigated on two

364 muscles, so the results cannot be extrapolated to other muscles or joint positions. Nevertheless,

365 this model may serve as an effective tool for generating hypotheses that may inform

366 experimental research with biomechanical implications.

\section{Acknowledgements}

368 We would like to thank Professor Stuart Phillips, for providing the position-CSA data necessary 369 to complete this model, and Dr. Silvia Blemker for reviewing and critiquing an early rendition of 370 our model. 
371

372

373

374

375

376

377

378

379

380

381

382

383

384

385

386

387

388

389

390

391

392

393

394

395

396

397

398

399

400

401

402

403

404

405

406

407

408

409

410

411

412

413

414

415

416

\section{References}

Aagaard P, Andersen JL, Dyhre-Poulsen P, Leffers AM, Wagner A, Magnusson SP, HalkjaerKristensen J, and Simonsen EB. 2001. A mechanism for increased contractile strength of human pennate muscle in response to strength training: changes in muscle architecture. The Journal of physiology 534:613-623. 10.1111/j.14697793.2001.t01-1-00613.x

Abbott BC, and Wilkie DR. 1953. The relation between velocity of shortening and the tension-length curve of skeletal muscle. Journal of Physiology 120:214-223.

Akagi R, Iwanuma S, Hashizume S, Kanehisa H, Fukunaga T, and Kawakami Y. 2015. Determination of contraction-induced changes in elbow flexor cross-sectional area for evaluating muscle size-strength relationship during contraction. Journal of Strength and Conditioning Research 29:1741-1747. 10.1519/JSC.0000000000000793

Amis A, Dowson D, and Wright V. 1979. Muscle strengths and musculoskeletal geometry of the upper limb. Engineering in Medicine 8:41-48.

An KN, Hui FC, Morrey BF, Linscheid RL, and Chao EY. 1981. Muscles across the elbow joint: a biomechanical analysis. Journal of Biomechanics 14:659-669.

Arnold P, and Bautmans I. 2014. The influence of strength training on muscle activation in elderly persons: a systematic review and meta-analysis. Experimental Gerontology 58:58-68. 10.1016/j.exger.2014.07.012

Baroni BM, Geremia JM, Rodrigues R, De Azevedo Franke R, Karamanidis K, and Vaz MA. 2013. Muscle architecture adaptations to knee extensor eccentric training: rectus femoris vs. vastus lateralis. Muscle and Nerve 48:498-506. 10.1002/mus.23785

Behm DG. 1995. Neuromuscular implications and applications of resistance training. Journal of Strength and Conditioning Research 9:264-274. 10.1097/00124278199511000-00014

Blazevich AJ, Gill ND, Bronks R, and Newton RU. 2003. Training-specific muscle architecture adaptation after 5-wk training in athletes. Medicine and Science in Sports and Exercise 35:2013-2022. 10.1249/01.MSS.0000099092.83611.20

Brandenburg JP, and Docherty D. 2002. The effects of accentuated eccentric loading on strength, muscle hypertrophy, and neural adaptations in trained individuals. Journal of Strength and Conditioning Research 16:25-32.

Brechue WF, and Abe T. 2002. The role of FFM accumulation and skeletal muscle architecture in powerlifting performance. European Journal of Applied Physiology 86:327-336.

Cage DJ, Abrams RA, Callahan JJ, and Botte MJ. 1995. Soft tissue attachments of the ulnar coronoid process. An anatomic study with radiographic correlation. Clinical Orthopaedics and Related Research:154-158.

Campos GE, Luecke TJ, Wendeln HK, Toma K, Hagerman FC, Murray TF, Ragg KE, Ratamess NA, Kraemer WJ, and Staron RS. 2002. Muscular adaptations in response to three different resistance-training regimens: specificity of repetition maximum training zones. European Journal of Applied Physiology 88:50-60. 10.1007/s00421-002-06816

de Souza EO, Tricoli V, Aoki MS, Roschel H, Brum PC, Bacurau AV, Silva-Batista C, Wilson JM, Neves M, Jr., Soares AG, and Ugrinowitsch C. 2014. Effects of concurrent strength and endurance training on genes related to myostatin signaling pathway and muscle 
417

418

419

420

421

422

423

424

425

426

427

428

429

430

431

432

433

434

435

436

437

438

439

440

441

442

443

444

445

446

447

448

449

450

451

452

453

454

455

456

457

458

459

460

461

462

fiber responses. Journal of Strength and Conditioning Research 28:3215-3223. 10.1519/JSC.0000000000000525

Dirks M, Wall B, Goossens G, van de Valk B, and van Loon L. 2015. One Week of Bed-rest Substantially Reduces Muscle Mass and Induces Insulin Resistance in Healthy, Young Males. The FASEB Journal 29:825.827.

Edgerton VR, Apor P, and Roy RR. 1990. Specific tension of human elbow flexor muscles. Acta Physiologica Hungarica 75:205-216.

Ekblom MM. 2010. Improvements in dynamic plantar flexor strength after resistance training are associated with increased voluntary activation and V-to-M ratio. Journal of Applied Physiology 109:19-26. 10.1152/japplphysiol.01307.2009

Erskine RM, Fletcher G, and Folland JP. 2014. The contribution of muscle hypertrophy to strength changes following resistance training. European Journal of Applied Physiology 114:1239-1249. 10.1007/s00421-014-2855-4

Erskine RM, Jones DA, Maffulli N, Williams AG, Stewart CE, and Degens H. 2011. What causes in vivo muscle specific tension to increase following resistance training? Experimental Physiology 96:145-155. 10.1113/expphysiol.2010.053975

Erskine RM, Jones DA, Williams AG, Stewart CE, and Degens H. 2010a. Inter-individual variability in the adaptation of human muscle specific tension to progressive resistance training. European Journal of Applied Physiology 110:1117-1125. 10.1007/s00421-010-1601-9

Erskine RM, Jones DA, Williams AG, Stewart CE, and Degens H. 2010b. Resistance training increases in vivo quadriceps femoris muscle specific tension in young men. Acta Physiologica (Oxford, England) 199:83-89. 10.1111/j.1748-1716.2010.02085.x

Gasser H, and Hill A. 1924. The Dynamics of Muscular Contraction. Proceedings of the Royal Society of London B: Biological Sciences 96:398-437.

Greening N, Harvey-Dunstan T, Williams J, Morgan M, Singh S, and Steiner M. 2014. Lower limb muscle mass using ultrasound predicts re-hospitalisation following admission for acute exacerbations of chronic respiratory disease. European Respiratory Journal 44:417.

Harber M, and Trappe S. 2008. Single muscle fiber contractile properties of young competitive distance runners. Journal of Applied Physiology 105:629-636. 10.1152/japplphysiol.00995.2007

Hill A. 1922. THE MECHANISM OF MUSCULAR CONTRACTION. Physiological Reviews 2:310341.

Hill AV. 1938. The Heat of Shortening and the Dynamic Constants of Muscle. Proceedings of the Royal Society B: Biological Sciences 126. 10.1098/rspb.1938.0050

Huijing PA, and Jaspers RT. 2005. Adaptation of muscle size and myofascial force transmission: a review and some new experimental results. Scandinavian Journal of Medicine and Science in Sports 15:349-380. 10.1111/j.1600-0838.2005.00457.x

Ikai M, and Fukunaga T. 1968. Calculation of muscle strength per unit cross-sectional area of human muscle by means of ultrasonic measurement. Internationale Zeitschrift für Angewandte Physiologie Einschliesslich Arbeitsphysiologie 26:26-32.

Ikegawa S, Funato K, Tsunoda N, Kanehisa H, Fukunaga T, and Kawakami Y. 2007. Muscle force per cross-sectional area is inversely related with pennation angle in strength trained athletes. Journal of Strength and Conditioning Research 22:128-131. 10.1519/JSC.0b013e31815f2fd3 
463 Ito M, Kawakami Y, Ichinose Y, Fukashiro S, and Fukunaga T. 1998. Nonisometric behavior 464 of fascicles during isometric contractions of a human muscle. Journal of Applied $465 \quad$ Physiology 85:1230-1235.

466 Janssen I, Baumgartner RN, Ross R, Rosenberg IH, and Roubenoff R. 2004. Skeletal muscle

467

468

469

470

471

472

473

474

475

476

477

478

479

480

481

482

483

484

485

486

487

488

489

490

491

492

493

494

495

496

497

498

499

500

501

502

503

504

505

506

507

508 cutpoints associated with elevated physical disability risk in older men and women. American Journal of Epidemiology 159:413-421.

Kawakami Y, Abe T, and Fukunaga T. 1993. Muscle-fiber pennation angles are greater in hypertrophied than in normal muscles. Journal of Applied Physiology 74:2740-2744.

Kawakami Y, Abe T, Kuno SY, and Fukunaga T. 1995. Training-induced changes in muscle architecture and specific tension. European Journal of Applied Physiology and Occupational Physiology 72:37-43.

Kawakami Y, Nakazawa K, Fujimoto T, Nozaki D, Miyashita M, and Fukunaga T. 1994. Specific tension of elbow flexor and extensor muscles based on magnetic resonance imaging. European Journal of Applied Physiology and Occupational Physiology 68:139-147.

Klass M, Baudry S, and Duchateau J. 2007. Voluntary activation during maximal contraction with advancing age: a brief review. European Journal of Applied Physiology 100:543551. 10.1007/s00421-006-0205-X

Maeo S, Takahashi T, Takai Y, and Kanehisa H. 2013. Trainability of muscular activity level during maximal voluntary co-contraction: comparison between bodybuilders and nonathletes. PloS One 8:e79486. 10.1371/journal.pone.0079486

Maeo S, Yoshitake Y, Takai Y, Fukunaga T, and Kanehisa H. 2014. Effect of short-term maximal voluntary co-contraction training on neuromuscular function. International Journal of Sports Medicine 35:125-134. 10.1055/s-0033-1349137

Malkov S, Cawthon PM, Peters KW, Cauley JA, Murphy RA, Visser M, Wilson JP, Harris T, Satterfield S, Cummings S, Shepherd JA, and Health ABCS. 2015. Hip Fractures Risk in Older Men and Women Associated With DXA-Derived Measures of Thigh Subcutaneous Fat Thickness, Cross-Sectional Muscle Area, and Muscle Density. Journal of Bone and Mineral Research 30:1414-1421. 10.1002/jbmr.2469

Mazzocca AD, Cohen M, Berkson E, Nicholson G, Carofino BC, Arciero R, and Romeo AA. 2006. The anatomy of the bicipital tuberosity and distal biceps tendon. Journal of shoulder and elbow surgery / American Shoulder and Elbow Surgeons [et al] 16:122127. 10.1016/j.jse.2006.04.012

Murray WM, Buchanan TS, and Delp SL. 2002. Scaling of peak moment arms of elbow muscles with upper extremity bone dimensions. Journal of Biomechanics 35:19-26.

Murray WM, Delp SL, and Buchanan TS. 1995. Variation of muscle moment arms with elbow and forearm position. Journal of Biomechanics 28:513-525.

Nagano A, and Komura T. 2003. Longer moment arm results in smaller joint moment development, power and work outputs in fast motions. Journal of Biomechanics 36:1675-1681.

Narici MV, and Kayser B. 1995. Hypertrophic response of human skeletal muscle to strength training in hypoxia and normoxia. European Journal of Applied Physiology and Occupational Physiology 70:213-219.

Nygaard E, Houston M, Suzuki Y, Jorgensen K, and Saltin B. 1983. Morphology of the brachial biceps muscle and elbow flexion in man. Acta Physiologica Scandinavica 117:287-292. 10.1111/j.1748-1716.1983.tb07208.x 
509

510

511

512

513

514

515

516

517

518

519

520

521

522

523

524

525

526

527

528

529

530

531

532

533

534

535

536

537

538

539

540

541

542

543

544

545

546

547

548

549

550

551

552

553

554
Nyitrai M, Rossi R, Adamek N, Pellegrino MA, Bottinelli R, and Geeves MA. 2006. What limits the velocity of fast-skeletal muscle contraction in mammals? Journal of Molecular Biology 355:432-442. 10.1016/j.jmb.2005.10.063

Pansarasa O, Rinaldi C, Parente V, Miotti D, Capodaglio P, and Bottinelli R. 2009. Resistance training of long duration modulates force and unloaded shortening velocity of single muscle fibres of young women. Journal of Electromyography and Kinesiology 19:e290-300. 10.1016/j.jelekin.2008.07.007

Parente V, D'Antona G, Adami R, Miotti D, Capodaglio P, De Vito G, and Bottinelli R. 2008. Long-term resistance training improves force and unloaded shortening velocity of single muscle fibres of elderly women. European Journal of Applied Physiology 104:885-893. 10.1007/s00421-008-0845-0

Pauwels F. 1980. Biomechanics of the locomotor apparatus: contribution on the functional anatomy of the locomotor apparatus. Berlin: Spfinger-Verlag.

Piazzesi G, Reconditi M, Linari M, Lucii L, Bianco P, Brunello E, Decostre V, Stewart A, Gore DB, Irving TC, Irving M, and Lombardi V. 2007. Skeletal muscle performance determined by modulation of number of myosin motors rather than motor force or stroke size. Cell 131:784-795. 10.1016/j.cell.2007.09.045

Power GA, Makrakos DP, Stevens DE, Rice CL, and Vandervoort AA. 2015. Velocity dependence of eccentric strength in young and old men: the need for speed! Applied Physiology, Nutrition, and Metabolism Physiologie Appliquée, Nutrition et Métabolisme 40:703-710. 10.1139/apnm-2014-0543

Ralston HJ, Polissar MJ, and et al. 1949. Dynamic features of human isolated voluntary muscle in isometric and free contractions. Journal of Applied Physiology 1:526-533.

Ramsay JW, Hunter BV, and Gonzalez RV. 2009. Muscle moment arm and normalized moment contributions as reference data for musculoskeletal elbow and wrist joint models. Journal of Biomechanics 42:463-473. 10.1016/j.jbiomech.2008.11.035

Reeves ND, Maganaris CN, Longo S, and Narici MV. 2009. Differential adaptations to eccentric versus conventional resistance training in older humans. Experimental Physiology 94:825-833. 10.1113/expphysiol.2009.046599

Riley DA, Bain JL, Thompson JL, Fitts RH, Widrick JJ, Trappe SW, Trappe TA, and Costill DL. 1998. Disproportionate loss of thin filaments in human soleus muscle after 17-day bed rest. Muscle and Nerve 21:1280-1289.

Sale DG, Martin JE, and Moroz DE. 1992. Hypertrophy without increased isometric strength after weight training. European Journal of Applied Physiology and Occupational Physiology 64:51-55.

Schuenke MD, Herman JR, Gliders RM, Hagerman FC, Hikida RS, Rana SR, Ragg KE, and Staron RS. 2012. Early-phase muscular adaptations in response to slow-speed versus traditional resistance-training regimens. European Journal of Applied Physiology 112:3585-3595. 10.1007/s00421-012-2339-3

Seynnes OR, de Boer M, and Narici MV. 2006. Early skeletal muscle hypertrophy and architectural changes in response to high-intensity resistance training. Journal of applied physiology (Bethesda, Md : 1985) 102:368-373. 10.1152/japplphysiol.00789.2006

Shiba R, Sorbie C, Siu DW, Bryant JT, Cooke TD, and Wevers HW. 1987. Geometry of the humeroulnar joint. Journal of Orthopaedic Research 6:897-906. 10.1002/jor.1100060614 
555

556

557

558

559

560

561

562

563

564

565

566

567

568

569

570

571

572

573

574

575

576

577

578

579

580

581

582

583

584

585

586

587

588

589

590

591

592

593

594

595

596

597

598

599

Siahkouhian M, and Hedayatneja M. 2010. Correlations of anthropometric and body composition variables with the performance of young elite weightlifters. Journal of Human Kinetics 25:125-131.

Srikanthan P, and Karlamangla AS. 2014. Muscle mass index as a predictor of longevity in older adults. American Journal of Medicine 127:547-553.

10.1016/j.amjmed.2014.02.007

Staron RS, Leonardi MJ, Karapondo DL, Malicky ES, Falkel JE, Hagerman FC, and Hikida RS. 1991. Strength and skeletal muscle adaptations in heavy-resistance-trained women after detraining and retraining. Journal of Applied Physiology 70:631-640.

Streja E, Molnar MZ, Kovesdy CP, Bunnapradist S, Jing J, Nissenson AR, Mucsi I, Danovitch GM, and Kalantar-Zadeh K. 2011. Associations of pretransplant weight and muscle mass with mortality in renal transplant recipients. Clinical Journal of the American Society of Nephrology 6:1463-1473. 10.2215/CJN.09131010

Sugisaki N, Wakahara T, Miyamoto N, Murata K, Kanehisa H, Kawakami Y, and Fukunaga T. 2010. Influence of muscle anatomical cross-sectional area on the moment arm length of the triceps brachii muscle at the elbow joint. Journal of Biomechanics. 10.1016/j.jbiomech.2010.06.013

Sugisaki N, Wakahara T, Murata K, Miyamoto N, Kawakami Y, Kanehisa H, and Fukunaga T. 2014. Influence of Muscle Hypertrophy on the Moment Arm of the Triceps Brachii Muscle. Journal of Applied Biomechanics. 10.1123/jab.2014-0126

Takarada Y, Takazawa H, Sato Y, Takebayashi S, Tanaka Y, and Ishii N. 2000. Effects of resistance exercise combined with moderate vascular occlusion on muscular function in humans. Journal of Applied Physiology 88:2097-2106.

van der Linden BJ, Koopman HF, Grootenboer HJ, and Huijing PA. 1998. Modelling functional effects of muscle geometry. Journal of Electromyography and Kinesiology 8:101-109.

Venturelli M, Saggin P, Muti E, Naro F, Cancellara L, Toniolo L, Tarperi C, Calabria E, Richardson RS, Reggiani C, and Schena F. 2015. In vivo and in vitro evidence that intrinsic upper- and lower-limb skeletal muscle function is unaffected by ageing and disuse in oldest-old humans. Acta Physiologica (Oxford, England). 10.1111/apha.12524

Vikne H, Refsnes PE, Ekmark M, Medbo JI, Gundersen V, and Gundersen K. 2006. Muscular performance after concentric and eccentric exercise in trained men. Medicine and Science in Sports and Exercise 38:1770-1781. 10.1249/01.mss.0000229568.17284.ab

Weijs PJ, Looijaard WG, Dekker IM, Stapel SN, Girbes AR, Oudemans-van Straaten HM, and Beishuizen A. 2014. Low skeletal muscle area is a risk factor for mortality in mechanically ventilated critically ill patients. Critical Care (London, England) 18:R12. 10.1186/cc13189

West DW, Burd NA, Tang JE, Moore DR, Staples AW, Holwerda AM, Baker SK, and Phillips SM. 2010. Elevations in ostensibly anabolic hormones with resistance exercise enhance neither training-induced muscle hypertrophy nor strength of the elbow flexors. Journal of Applied Physiology 108:60-67. 10.1152/japplphysiol.01147.2009

West DWD, Burd NA, Tang JE, Moore DR, Staples AW, Holwerda AM, Baker SK, and Phillips SM. 2009. Elevations in ostensibly anabolic hormones with resistance exercise 
600 enhance neither training-induced muscle hypertrophy nor strength of the elbow $601 \quad$ flexors. Journal of Applied Physiology 108. 10.1152/japplphysiol.01147.2009

602 Winwood PW, Keogh JW, and Harris NK. 2012. Interrelationships between strength,

603 anthropometrics, and strongman performance in novice strongman athletes. Journal $604 \quad$ of Strength and Conditioning Research 26:513-522.

$605 \quad$ 10.1519/JSC.0b013e318220db1a

606 


\section{Table $\mathbf{1}$ (on next page)}

Anatomical cross-sectional areas, moment arm lengths, and moment contributions.

Taking into account the changing moment arm broadens the elbow flexion moment contribution range of both the biceps brachii and brachialis. 
1

\begin{tabular}{|l|c|c|c|c|c|}
\hline & Atrophy & $\Delta_{\mathrm{AB}}$ & Baseline & $\Delta_{\mathrm{HB}}$ & Hypertrophy \\
\hline BIC ACSA $\left(\mathrm{cm}^{2}\right)$ & 5.50 & -5.50 & 11.00 & +11.00 & 22.00 \\
\hline BRA ACSA $\left(\mathrm{cm}^{2}\right)$ & 5.50 & -5.50 & 11.00 & +11.00 & 22.00 \\
\hline BIC MA length (cm) & 1.63 & -0.54 & 2.17 & +0.59 & 2.76 \\
\hline BRA MA length (cm) & 0.54 & -0.21 & 0.75 & +0.28 & 1.03 \\
\hline BIC moment contribution (N·m) (incl. MA) & 2.73 & -4.34 & 7.07 & +9.10 & 16.17 \\
\hline BRA moment contribution $(\mathrm{N} \cdot \mathrm{m})$ (incl. MA) & 0.9 & -1.59 & 2.49 & +4.05 & 6.54 \\
\hline BIC moment contribution (N·m) (excl. MA) & 3.63 & -3.44 & 7.07 & +5.62 & 12.69 \\
\hline BRA moment contribution (N.m) (excl. MA) & 1.26 & -1.23 & 2.49 & +2.27 & 4.76 \\
\hline
\end{tabular}

2 


\section{Figure 1 (on next page)}

Graphical visualization of the model.

$x$ represents the proximal distance from the elbow joint. The muscle bellies end at the point where the tangent line, $t$, representative of the tendon, would insert into the appropriate location distal to the elbow. Moment arms were calculated as the perpendicular distance from the joint center (origin) to the tendon $t$. Parameters for $t$ represent the position proximal to the elbow, coefficient of hypertrophy, $x_{\max }$, and anterior/superficial position. Parameters for $B R A$ and $B I C$ represent the position proximal to the elbow and coefficient of hypertrophy. The parameter of $r$ simply represents the position proximal to the elbow. 


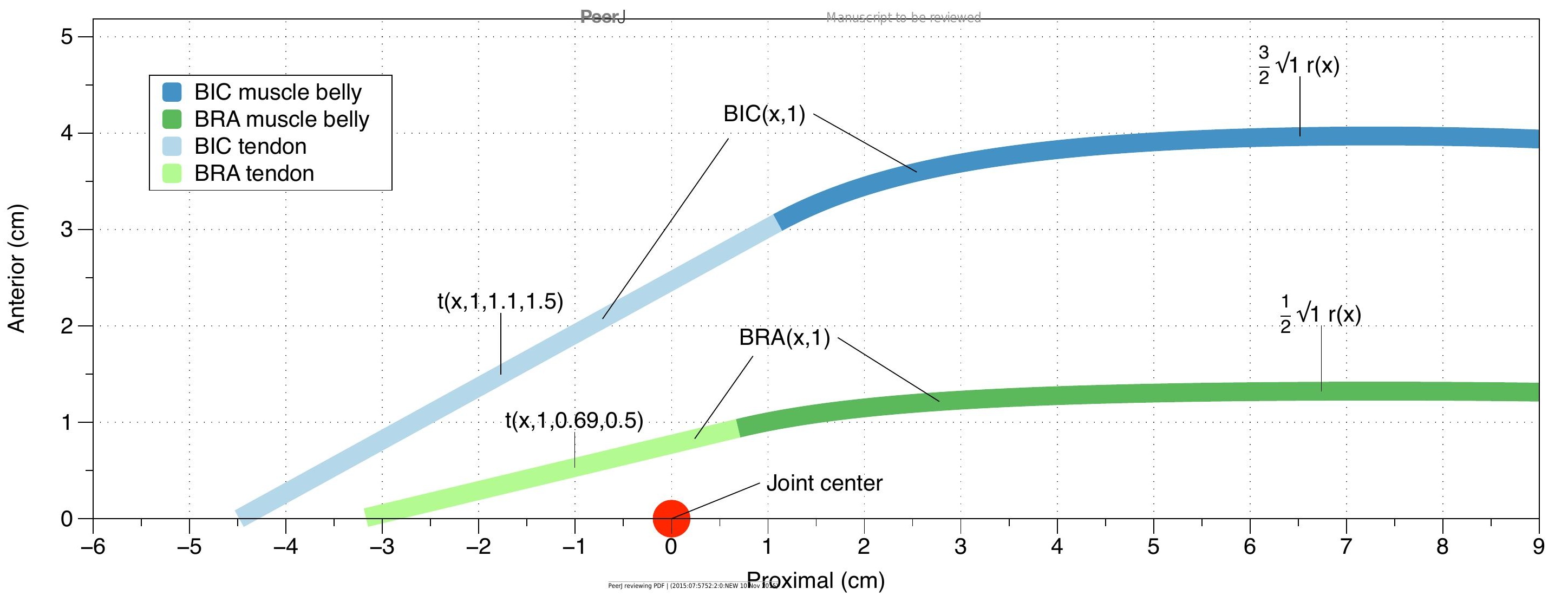




\section{Figure 2 (on next page)}

Biomechanical variables as a function of anatomical cross-sectional area.

(A) Relationship between biceps brachii anatomical cross-sectional area and muscle moment arm. Negatively sloped lines are normal BIC MAs, and positively sloped lines are empirical BRA MAs (Ramsay et al. 2009). (B) Pennation angles of the BIC and BRA increase linearly with increases in ACSA. (C) Muscle force increases with ACSA. (BIC plots are underneath, and identical to, the BRA plots.) (D) Tendon force increases with ACSA. (E) The elbow flexion moment contributions of the BIC and BRA with changes in ACSA, both with and without the changes in MA length. Vertical lines at $11.0 \mathrm{~cm}^{2}$ indicate baseline. 


\section{Figure 3 (on next page)}

Illustration of the changes in biceps brachii and brachialis moment arm lengths with increases in anatomical cross-sectional area.

By doubling the anatomical cross-sectional area of the biceps brachii and brachialis, the moment arms of each increase by $27.2 \%$ and $37.3 \%$, respectively. 
